\title{
Comparação de sistemas de produção de pitaya (Hylocereus Costaricensis) com diferentes níveis tecnológicos na Amazônia brasileira
}

O artigo objetivou analisar a sensibilidade da produção de pitaya (Hylocereus costaricensis) fertirrigada no município de Tomé-Açu/PA. A coleta de dados deu-se através de entrevistas com produtores locais para levantar informações sobre os custos de produção de um hectare $\left(10.000 \mathrm{~m}^{2}\right)$ e compreender as características dos principais canais de comercialização da produção. Para análise dos dados, usou-se o software Excel para a elaboração de fluxos de caixa, cálculo de indicadores de viabilidade econômica e realização de análise da sensibilidade dos custos e preços no intervalo de 2016 a 2018, além de realizar projeções para o ano de 2019 com base na Média Móvel Ponderada (MMP). Na comparação entre o primeiro e o último ano, identificou-se o aumento dos custos de produção, assim como a queda no preço de comercialização no canal principal. Constatou-se, também, um aumento de mais de $23 \%$ no valor de investimento inicial e queda da MCU, além do acréscimo no tempo de retorno do investimento. As oscilações nos preços impactaram consideravelmente a rentabilidade da produção no período de 2016 a 2018 e o valor projetado para 2019, sendo que a principal diferença ocorreu entre os anos de 2017 a 2018 , nos quais o VPL, a uma taxa desconto de $10 \%$ a.a. apresentou retração de $\mathrm{R} \$ 67.333,82$. Observou-se, no entanto, que os indicadores se mostraram viáveis em todos os períodos observados e projetados, com o VPL possuindo resultados positivos em todos os cenários - o IBC $>1$ e a TIR $>$ TMA - , comprovando a viabilidade econômica da atividade.

\section{Comparison of pitaya (Hylocereus Costaricensis) production systems with different technological levels in the Brazilian Amazon}

\begin{abstract}
The article aimed analyze the sensitivity of production of pitaya (Hylocereus costaricensis) fertigated in the municipality of Tomé-Açu/PA. Data collection took place through interviews with local producers to gather information about the production costs of 1 (one) ha (hectare) and to understand the characteristics of the main production marketing channels. For data analysis, Excel software was used to prepare cash flows, calculate economic viability indicators, and carry out analysis of the sensitivity of costs and prices in the range from 2016 to 2018, in addition to making projections for the year 2019 based on in the Weighted Moving Average (MMP). In the comparison between the first and the last year, an increase in production costs was identified, as well as a drop in the price of marketing in the main marketing channel. There was also an increase of more than $23 \%$ in the initial investment value, and a decrease in the MCU, in addition to an increase in the return on investment. The price fluctuations considerably impacted the production profitability, in the period from 2016 to 2018 and in the projected value for 2019, with the main difference occurring between the years 2017 to 2018 , where the NPV at a discount rate of $10 \%$ pa, presented a retraction of R\$ $67,333.82$. However, it was observed that the indicators proved to be viable in all the observed and projected periods, with NPV having positive results in all scenarios, the $\mathrm{IBC}>1$, the TIR>TMA, proving the economic viability of the activity.
\end{abstract}

Keywords: Economic viability; Economic sensitivity; Sales channels.

Topic: Extensão e Desenvolvimento Rural

Reviewed anonymously in the process of blind peer.

João Paulo Borges de Loureiro (ic

Universidade Federal Rural da Amazônia, Brasil

http://lattes.cnpq.br/0417040128680889

http://orcid.org/0000-0001-8586-8201

joaopaulo_loureiro@hotmail.com

Adriana Paiva dos Praseres Pires

Universidade Federal Rural da Amazônia, Brasil

http://lattes.cnpq.br/0072624585524132

http://orcid.org/0000-0001-8607-6548

adrianapaiva21@hotmail.com

Marcos Antônio Souza dos Santos (iD

Universidade Federal Rural da Amazônia, Brasil

http://lattes.cnpq.br/1517009704490133

http://orcid.org/0000-0003-1028-1515

marcos.marituba@gmail.com

6

DOI: 10.6008/CBPC2179-6858.2021.006.0010
Received: 08/06/2021

Approved: 24/06/2021
Fabrício Khoury Rebello (10)

Universidade Federal Rural da Amazônia, Brasil

http://lattes.cnpq.br/8656930211054464

http://orcid.org/0000-0003-2398-4906

fabriciorebello@hotmail.com
Referencing this:

LOUREIRO, J. P. B.; PIRES, A. P. P.; SANTOS, M. A. S.; REBELLO, F. K. Comparação de sistemas de produção de pitaya (Hylocereus Costaricensis) com diferentes níveis tecnológicos na Amazônia brasileira. Revista Ibero Americana de Ciências Ambientais, v.12, n.6, p.118-129, 2021. DOI: http://doi.org/10.6008/CBPC21796858.2021.006.0010 


\section{INTRODUÇÃO}

O cultivo da pitaya vem ganhando cada vez mais adeptos nos últimos anos, sendo que Le Bellec et al. (2006) afirmam que os diversos tipos de pitaya são agrupados em quatro gêneros - Stenocereus, Cereus, Selenicereus e Hylocereus -, sendo as mais conhecidas e cultivadas a pitaya amarela (Selenicereus megalanthus), que tem casca amarela e polpa branca, e a pitaya vermelha, do gênero Hylocereus, com a casca vermelha e a polpa branca ou vermelha, dependendo da espécie. As pitayas são nativas das Américas e são também cultivadas na Colômbia, México, Israel e Vietnã, países considerados potenciais produtores mundiais.

Atualmente, no Brasil, essa fruticultura é pouco estudada, existem trabalhos pontuais e específicos. Cavalcante et al. (2006) dizem que há a necessidade de estudos mais abrangentes sobre aspectos práticos e de informar ao potencial produtor as tecnologias que devem ser aplicadas sob as condições brasileiras. Na mesma vertente, Sato et al. (2014) declaram que, por ser pouco conhecida, estudos relacionados à viabilidade econômica e a termos físico-químicos desse cultivo ainda são incipientes, evidenciando, assim, a importância de se desenvolver estudos nessas vertentes em diferentes territórios brasileiros.

Aos poucos, a pitaya vem se destacando no mercado com uma boa aceitação devido aos seus atributos nutritivos: é composta por vitaminas e minerais que funcionam como antioxidantes, os quais auxiliam na imunização do organismo, manutenção dos ossos e dentes e na produção de células vermelhas. Assim, também por seu sabor, cor e textura, o fruto é considerado um "superalimento". Ademais, como afirma Donadio (2009), a pitaya destaca-se pela probabilidade de comercialização, portanto a tendência é o crescente aumento da produção para os anos posteriores.

O consumo dela pode ocorrer de maneira diversa, natural ou processada, na fabricação de sucos, doces, sorvetes, geleias, entre outros. Essa variedade de usos permite grande aprovação dos mercados consumidores. A pitaya vem ganhando espaço de destaque na produção agrícola brasileira por ser considerada novidade e, principalmente, por ser uma cultura de rápido retorno, pois é produzida logo no primeiro ano após o plantio (JUNQUEIRA et al., 2002).

No estado do Pará, a pitaya vem sendo cultivada em vários municípios e o de Tomé-Açu apresentase como um dos principais produtores. Em razão do proeminente potencial do fruto para o mercado interno e externo, ele é facilmente encontrado nas propriedades rurais da região.

Sabe-se que o início do cultivo desse fruto no município era voltado ao consumo próprio, à degustação e ao escambo com outros produtos agrícolas entre os agricultores. Hoje, a localidade do Breu, 25.000 m distante do centro de Tomé-Açu, é a maior região produtora do fruto (CAMTA, 2018). Ressalta-se que a produção da pitaya teve seu despontamento em escala comercial por volta de 2012, período em que as pitayas eram vistas nas feiras e gôndolas, alcançando, dessa forma, o mercado varejista.

Em geral, o fruto é comercializado em feiras livres, ou direcionado como commodity - produto de origem primária - às empresas beneficiadoras. Baseado nos dados da Central de Abastecimento do Pará S/A (CEASA/PA), em 2011 foram vendidos 13.194 kg de pitayas com origem apenas do município de Tomé- 
Açu. Os preços de comercialização do quilo, no primeiro semestre de 2017 , variaram de $\mathrm{R} \$ 6,00$ a $\mathrm{R} \$ 8,00$ (CAMTA, 2018).

Apesar do crescimento na demanda, e embora seja considerada uma cultura rentável, é de suma importância a realização de pesquisas expondo não somente resultados referentes à genética e ao manejo, mas também que analisem economicamente a cultura na região de Tomé-Açu, considerando diversos fatores. Em virtude dos escassos estudos sobre essa cultura, sob a ótica econômica, o objetivo do presente trabalho foi analisar a sensibilidade da produção de pitaya (Hylocereus costaricensis) produzida em ToméAçu e comercializada nos diferentes canais que os produtores do município têm acesso e, a partir desse enfoque, determinar o quanto esses cenários influenciam na rentabilidade da produção.

\section{REVISÃO TEÓRICA}

\section{Contextualização sobre o cultivo da pitaya}

A palavra pitaya, que significa "fruta escamosa", é de origem do idioma Taíno, língua falada na região das Ilhas Antilhas na época da conquista espanhola, no século XV (NUNES et al., 2014). Pelo mundo, a espécie possui várias nomenclaturas: Pitaya, Pitajaja, Night blooming, Flor de Cáliz, Tasojo, Vine Cacti e Dragon Fruit, como é mais comumente conhecida (LE BELLEC et al., 2006).

Essa cactácea, nativa da região das Américas (GARCÍA-RUBIO et al., 2015), especificamente da América Central, é frequentemente cultivada em nível de agricultura familiar, principalmente em cultivos agroecológicos, utilizada na medicina popular e como importante fonte de alimento (CRUZ et al., 2015). Algumas décadas atrás, essa frutífera era pouco conhecida, mas hoje ocupa um importante nicho no mercado mundial de frutas exóticas, principalmente por conta de suas características.

A pitaya é resistente à seca e pouco exigente quanto à qualidade do solo. Espinhosa, subsiste em condições naturais limitantes e apresenta vasta gama de características anatômicas e fisiológicas para conservar água. Botanicamente, deriva das epífitas e da subfamília Cereoideae, que inclui o gênero Cereus dentro deste, deriva das espécies Cereus undatus, Cereus polyrhizus e Cereus megalanthus, esta última pode ser encontrada como sinônimo de Hylocereus (CANTO et al., 1993). Na produção agrícola comercial, porém, destacam-se quatro diferentes espécies, as quais representam uma possibilidade de diversificação agrícola: Hylocereus undatus, de casca vermelha e polpa branca; Hylocereus costaricensis, de casca vermelha e polpa roxa; Hylocereus polyrhizus, de casca vermelha e polpa vermelha; e Selenicereus megalanthus, de casca amarela e polpa branca (JUNQUEIRA et al., 2002).

No que concerne ao cultivo comercial, atualmente a pitaya é cultivada em diversos países de diferentes continentes, como: Austrália, Camboja, Colômbia, Equador, Guatemala, Indonésia, Israel, Nova Zelândia, Nicarágua, México, Peru, Filipinas, Taiwan, Tailândia, Estados Unidos, Vietnã e Brasil (MIZRAHI et al., 1997).

Como cacto epífito, a pitaya necessita de um tutor para seu crescimento, que pode ser árvores, mourões de madeira, postes de concreto, cercas, muros ou outras bases. Como a vida útil da planta é de 
cerca de vinte anos, a durabilidade do suporte também se apresenta importante nesse contexto, pois deve ser resistente, com intuito de suportar grande peso (GUNASENA et al., 2007) sendo que, no que diz respeito à produtividade, Vaillant et al. (2005) afirmaram que na Nicarágua cultivos bem conduzidos podem produzir até $26.000 \mathrm{~kg}$ por hectare e, após cinco anos, apresentam produtividade de $12.000 \mathrm{~kg}$ por hectare (BASTOS et al., 2006).

A planta, de forma geral, apresenta-se como alternativa múltipla de usos. Do ponto de vista nutricional, a pitaya contém muita umidade, e, juntamente com outros alimentos, pode auxiliar para uma dieta equilibrada. Mas, o que chama atenção para inúmeros estudos é a quantidade de compostos bioativos, principalmente compostos fenólicos, tais como vitaminas e pigmentos (ABREU et al., 2012).

A maioria das pitayas é consumida ao natural, mas a polpa congelada pode ser utilizada para fazer sorvetes, iogurtes, geleias, conservas, compotas, sucos, doces, bolos, entre outros. Além do uso alimentício, o fruto tem sido alvo de estudos voltados à indústria farmacêutica e cosmética e ao tratamento de acne, queimaduras e caspa. Pode ser também processada e usada em produtos como sabonetes, cremes, hidratantes, loções, perfumes, pomadas etc., alcançando visibilidade internacional.

Como vantagem produtiva, as pitayas do gênero Hylocereus podem ser cultivadas em todos os continentes, em regiões de clima tropical e temperado, com manejos que vão da rusticidade à tecnificação do processo (LE BELLEC et al., 2006). A aquisição de tecnologia de ponta e investimentos na área de pesquisas científicas por países como Malásia, Estados Unidos, China, Tailândia e Israel permitem o destaque dessas regiões na produção e, por conseguinte, o aumento do rendimento e melhoria da qualidade dos frutos.

De acordo com Marques (2012), o cultivo e a comercialização da pitaya no Brasil ainda são prematuros. Hoje, há cultivos comerciais em Minas Gerais, Paraná, Santa Catarina, Mato Grosso do Sul, Rio Grande do Norte, Ceará, Pernambuco e Pará, sendo que no Brasil, as pitayas passaram a ser cultivadas em escala comercial a partir da década de 1990 e, em razão do crescente mercado, vêm a cada ano ganhando mais espaço em todo o território nacional.

O mercado de frutas exóticas vem ganhando prestígio no Brasil, dentre as quais Junqueira et al. (2002) citam a pitaya como frutífera promissora pela rusticidade, pois apresenta poucas exigências nutricionais do solo. Outras características da cultura são a resistência à baixa disponibilidade hídrica, o manejo simples e o baixo custo.

No que diz respeito à adubação de pitaya, Cavalcante et al. (2006) declaram que ela é baseada no cultivo orgânico, adotando-se principalmente o uso de esterco. Nesse sentido, no Brasil, especificamente, pouco se sabe sobre adubação, mineral ou orgânica, ou sobre a nutrição mineral do fruto, o que demonstra a necessidade de estudos com essas finalidades que possam subsidiar os atuais e potenciais produtores de pitaya, visto que a cultura se encontra em expansão no país, com despontamento de novos cultivares e diferentes sistemas de manejo.

Burt et al. (1995) afirmam que a fertirrigação é o mais econômico e eficiente método de aplicação de fertilizantes, especialmente quando utilizado por meio de sistemas de irrigação localizada, pois possibilita aumentos substanciais na qualidade dos frutos e, por conseguinte, no valor comercial. Portanto, com a boa 
aceitação do fruto, o desenvolvimento e a comercialização da produção, a tecnologia, por meio da fertirrigação, apresenta-se como uma nova alternativa no cultivo da pitaya.

\section{Fluxo de caixa e indicadores de viabilidade}

Para Gitman (2010), o fluxo de caixa representa as movimentações de dinheiro, sendo estas de operações, investimentos e financiamentos realizados pelas instituições, essa classificação deve estar relacionada com intervalos de tempos. Conforme as análises do fluxo de caixa, os gestores podem tomar decisões mais precisas.

Os valores expostos no fluxo de caixa servem de base para o cálculo de indicadores que atestam a viabilidade econômica de uma atividade. Dentre esses indicadores, destaca-se o valor presente líquido (VPL), que, para Ponciano et al. (2004), representa uma variação que o caixa pode sofrer de acordo com o tempo, devendo-se levar em consideração a aplicação de taxas de juros e os somar algebricamente.

Outro importante indicador calculado por meio do fluxo de caixa é a taxa interna de retorno (TIR), que é definida como a taxa de desconto de um investimento que torna seu valor presente líquido nulo, ou seja, o qual faz com que o projeto pague o investimento inicial quando se considera o valor do dinheiro no tempo. Assim, informa-se o quanto a atividade econômica irá render em um determinando momento, ao lhe comparar com as taxas oferecidas no mercado (BROM et al., 2007).

Outros importantes indicadores de viabilidade econômica calculados por meio do fluxo de caixa e que complementam as informações do VPL e da TIR são: o índice benefício custo (IBC) que, atesta Clemente (1998), é um método que informa o quanto se ganha por cada unidade de capital investida; o Payback, que é o tempo de retorno do capital investido, sendo definido como espaço de tempo necessário para que a economia operacional se iguale ao valor do investimento inicial; e, por fim, o ponto de equilíbrio (PE), que, na visão de Clemente (1998), representa o número mínimo de produtos a ser produzido e vendido para cobrir os custos de produções.

\section{METODOLOGIA}

Para o desenvolvimento da pesquisa, primeiramente foi feito um levantamento de informações bibliográficas sobre as temáticas inerentes à realização do trabalho. Posteriormente realizou-se, entre os anos de 2016 e 2018, visitas a propriedades de produtores de pitaya do município de Tomé-açu, localizado no nordeste do Pará, estado da Amazônia brasileira, onde o processo produtivo era acompanhado e, por meio de entrevistas com os responsáveis, foram coletados dados de preços de venda praticados pelos canais de comercialização do município e foram mensurados os custos de produção da cultura, que originaram planilhas de fluxos de caixa sobre o que a cultura da pitaya proporcionou aos produtores durante todo período de observação. É importante salientar que, por meio da série histórica de preços, elaborou-se uma simulação, através do método da média móvel ponderada, do preço a ser praticado no ano de 2019.

Para atestar a viabilidade ou inviabilidade dos fluxos de caixa elaborados, aplicou-se os cálculos dos indicadores de viabilidade econômica do VPL, IBC, TIR, Payback e PE, sendo que os quais foram calculados a 
partir das seguintes equações expostas em Loureiro et al. (2019).

Na Equação 1, conforme o valor presente líquido (VPL), o ano zero de investimento ( $F C O)$, o ano 1 de investimento (FC1), o múltiplo indefinido de um fator $(n)$ e a taxa (i):

$V P L=F C 0+\frac{F C 1}{(1+i)^{1}}+\frac{F C}{(1+i)^{2}}+\cdots \frac{F C n}{(1+i)^{n}} \quad$ Equação (1)

$\mathrm{Na}$ Equação 2, conforme a Taxa Interna de Retorno (TIR), o lucro líquido (L.L) e o capital inicial (I):

$$
T I R=\left(\frac{L . L}{I}-1\right) \times 100 \quad \text { Equação (2) }
$$

Na Equação 3, conforme o Índice Benefício/Custo $(I B C)$, a somatória $(\Sigma)$, o fluxo de caixa $\left(C F_{j}\right)$ e o ano 0 de investimento $\left(C F_{0}\right)$ :

$$
I B C=\frac{\sum \frac{\left[C F_{j}\right]}{(1+i)^{j}}}{C F_{0}} \quad \text { Equação (3) }
$$

Na Equação 4, conforme o Payback ( $P B)$, a somatória ( $\Sigma)$, o horizonte do projeto (Tquando), o fluxo de caixa total $\left(C F_{T}\right)$ e o investimento inicial $\left(I_{0}\right)$ :

$$
P B=\text { Tquando } \sum_{I=0}^{T} C F_{T}=I_{0} \quad \text { Equação (4) }
$$

$\mathrm{Na}$ Equação 5, conforme o ponto de equilíbrio em quantidade produzida $(Q)$, o custo fixo $(C F)$, o preço $(P)$ e o custo variável médio ( $C V m e)$ :

$$
Q=\frac{C F}{(P-C V m e)} \quad \text { Equação (5) }
$$

Por fim, para atestar o grau de risco de se investir na atividade, após o cálculo dos indicadores, realizou-se uma análise de sensibilidade para os comparar. Os indicadores oscilaram considerando os diferentes cenários existentes nos anos observados em cada canal de comercialização existente no município.

\section{RESULTADOS E DISCUSSÃO}

Primeiramente, procurou-se fazer a caracterização do sistema de produção de pitaya em Tomé-Açu, tendo em conta que a tipologia do sistema de produção foi criada levando em consideração as características de tecnologia, de espaçamento e de manejo adotadas na área de pesquisa, como mostra o Quadro 1.

Quadro 1: Caracterização do Sistema de Produção (2018).

\begin{tabular}{|l|l|}
\hline Itens & Sistema de Produção \\
\hline Tecnologia & Sistema de Irrigação \\
\hline Espaçamento & $2,5 \mathrm{~m} \times 2,5 \mathrm{~m}$ \\
\hline Quantidade de Pés & 1.600 (Quadrado) \\
\hline Meio de Adubação & Via Fertirrigação \\
\hline Tipo de Adubo Principal & Fertilizante Sintético \\
\hline
\end{tabular}

\section{Mercado Local}

No que concerne ao mercado local e a suas formas de comercialização, os produtores possuem, atualmente, três canais como alternativas para escoar a produção: intermediários ou atravessadores, Cooperativa Agrícola Mista de Tomé-Açu (CAMTA) e CEASA. Cada uma das três opções possui características diferentes de funcionamento, preço e critérios para a compra do produto. 
Os atravessadores são comerciantes de outros municípios que vêm a Tomé-Açu em busca do fruto quando falta abastecimento em suas regiões, um canal inconstante que depende diretamente da falta de produção dos agricultores de outras regiões ou do aumento da demanda desses locais.

Já a CAMTA, cooperativa que é o canal mais próximo e seguro para o produtor de Tomé-Açu, compra a produção tanto dos cooperados quanto dos não cooperados, sendo que, apesar de ser o canal que oferece o menor preço, comporta a maior parte da quantidade produzida pelo agricultor local, pois necessita de abastecimento contínuo para sua agroindústria. Por fim, a CEASA, localizada em Belém/PA, demanda o pagamento do frete para a comercialização da frutose, caso não sejam todos vendidos, os frutos retornam ao produtor. Além disso, os preços dependem da quantidade total disponibilizada pelo produtor.

Como pode ser visto no Quadro 2, cada canal possui vantagens e desvantagens para o produtor, sendo que cada um tem interferência direta no preço pago pelo produto. Dependendo do perfil que o produtor possui ou da necessidade que estiver passando, ele pode optar por um dos canais de comercialização aqui apresentados. Poderá escolher um canal de mais segurança, com a desvantagem do preço menor, como é o caso da CAMTA; escolher um canal com um grau de risco maior, como é o caso do atravessador, que não tem demanda fixa; ou ainda, optar pela CEASA, onde se recebe um melhor preço, porém assumindo o custo logístico de deslocamento até a capital a fim de comercializar o produto.

Quadro 2: Caracterização dos canais de comercialização existentes no mercado de Tomé-Açu.

\begin{tabular}{|l|l|l|l|}
\hline Características & Atravessador & CAMTA & CEASA \\
\hline Fluxo de Fornecimento & Aleatório & Contínuo & Contínuo \\
\hline Preço & Médio & Baixo & Alto \\
\hline Proximidade & Perto & Perto & Distante \\
\hline
\end{tabular}

Apesar de ser um produto exótico e estar no conhecimento popular há pouco tempo, o aumento pela demanda da pitaya não acompanhou uma evolução de preço pago ao produtor em dois dos três canais de comercialização existentes em Tomé-Açu. Isso pode ser explicado pelo fato do fruto hoje ser bem mais difundido entre os produtores locais do que em 2016, aumentando assim a oferta do produto e controlando a elevação dos preços.

O aumento pela demanda do fruto pode ser comprovado pelos dados do relatório de compra de matéria-prima da CAMTA, o qual se observou um aumento de 34,52\% na compra de pitaya de 2016 para 2017. Porém, é importante salientar que, entre esses dois anos, o preço pago pelo quilo da pitaya, neste canal, reduziu (ver Quadro 3). Este fato fez alguns produtores buscarem alternativas para vender sua produção, por isso, de acordo com o mesmo relatório de compra de matéria-prima, em 2016, 44 cooperados forneciam pitaya para a cooperativa e, em 2017, esse número caiu para 39.

Quadro 3: Preços pagos pelo quilo da pitaya aos produtores nos três canais de comercialização. Valores previstos para 2019 com base no método da Média Móvel Ponderada (MMP).

\begin{tabular}{|l|l|l|l|l|}
\hline Canal & $\mathbf{2 0 1 6}$ & $\mathbf{2 0 1 7}$ & $\mathbf{2 0 1 8}$ & $\mathbf{2 0 1 9 *}$ \\
\hline Atravessador & $\mathrm{R} \$ 6,00$ & $\mathrm{R} \$ 6,00$ & $\mathrm{R} \$ 4,00$ & $\mathrm{R} \$ 5,00$ \\
\hline CAMTA & $\mathrm{R} \$ 4,00$ & $\mathrm{R} \$ 3,80$ & $\mathrm{R} \$ 3,40$ & $\mathrm{R} \$ 3,63$ \\
\hline CEASA & $\mathrm{R} \$ 6,00$ & $\mathrm{R} \$ 12,00$ & $\mathrm{R} \$ 13,00$ & $\mathrm{R} \$ 11,50$ \\
\hline
\end{tabular}




\section{Produtividade da Pitaya}

Dentro do paradigma de análise do tripé custo-volume-lucro, que, para Gitman (1999), é fundamental para a determinação da viabilidade econômica ou não de uma atividade, buscou-se elaborar a curva de produtividade da pitaya fertirrigada no município de Tomé-Açu, com base nos dados da propriedade pesquisada, visando identificar qual a quantidade produzida do fruto em quilo durante seu primeiro ano de produção e durante os demais anos de vida útil do plantio.

Ressalta-se que não foram encontrados dados, tanto na literatura quanto na área pesquisada, que comprovem a queda de produtividade da pitaya. Em vista disso, a queda foi considerada a partir da estabilidade, produtividade constante durante sua vida útil. Nota-se na Figura 1 que a produção se inicia com um total de $1.600 \mathrm{~kg}$ por hectare no primeiro ano, seguida de $8.000 \mathrm{~kg}$ por hectare no segundo e atinge estabilidade produtiva no terceiro ano com $16.000 \mathrm{~kg}$ por hectare.

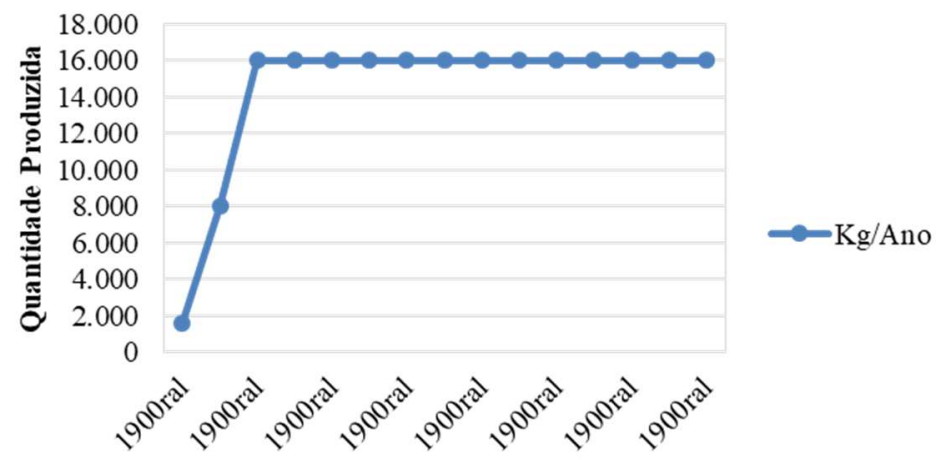

Figura 1: Curva de produtividade da pitaya fertirrigada.

\section{Resultado dos Indicadores de Viabilidade}

É importante salientar que os fluxos de caixa elaborados foram submetidos à ação de diferentes taxas de descontos utilizadas para constatar a viabilidade econômica da produção de pitaya fertirrigada, sendo que essas taxas são executadas nas principais linhas de financiamento que o produtor familiar do município tem acesso. Tais resultados foram calculados a partir da estabilidade produtiva que se dá no terceiro ano e considerando os custos fixos e variáveis necessários à produção.

Dentre as linhas de financiamento utilizadas, destacam-se o Programa de Agricultura de Baixo Carbono - ABC, com 5,5\% a.a.; o Mini FNO Rural, com 5,0\% a.a.; o PRONAF Mais Alimentos, com 2,0\% a.a.; e utilizada à taxa de 10,0\% a.a., simulando uma linha de crédito de um banco privado para configurar um cenário mais pessimista.

A importância do fluxo de caixa no processo de tomada de decisão foi relatada por Assaf Neto (2010), onde o mesmo é apresentado como um instrumento eficiente para a obtenção de financiamentos, tanto no presente como para o futuro. Tal visão é confirmada por Silva (2014), ao se referir ao fluxo de caixa como instrumento de tomada decisão de possíveis conquistas econômicas e financeiras de um projeto em variadas vertentes. 


\section{Cenário 01 - Ano 2016}

Com os valores obtidos no fluxo de caixa de 2016, foi possível encontrar os resultados dos indicadores de viabilidade econômica apresentados na Tabela 1, que comprovaram a viabilidade econômica e rentabilidade da produção de pitaya fertirrigada em Tomé-Açu, onde o VPL, considerando que a maior taxa de $10 \%$ a.a., foi de $\mathrm{R} \$ 214.933,57$.

Este valor refere-se ao lucro líquido ao final do projeto, uma vez que já foram descontados todos os custos gerados com a produção. Como um destaque, os resultados do IBC mostram que, para cada real investido na atividade, independente da taxa de desconto utilizada, o retorno é sempre acima de $R \$ 4,00$, confirmando a rentabilidade da cultura nesse sistema.

Tabela 1: Resultado dos indicadores de viabilidade com os custos de produção do ano de 2016.

\begin{tabular}{lllll}
\hline \multicolumn{2}{l}{ TAXAS DE FINANCIAMENTO } & & & \\
Cenário 01 - 2016 & $2,0 \%$ a.a. & $5,0 \%$ a.a. & $5,5 \%$ a.a. & $10 \%$ a.a. \\
\hline VPL & $\mathrm{R} \$ 457.246,17$ & $\mathrm{R} \$ 342.455,63$ & $\mathrm{R} \$ 326.632,39$ & $\mathrm{R} 214.933,57$ \\
$\mathrm{IBC}$ & $\mathrm{R} \$ 7,53$ & $\mathrm{R} \$ 5,89$ & $\mathrm{R} \$ 5,67$ & $\mathrm{R} \$ 4,07$ \\
TIR & $36,63 \%$ & $36,63 \%$ & $36,63 \%$ & $36,63 \%$ \\
PayBack & 3 anos e 4 meses & 3 anos e 4 meses & 3 anos e 4 meses & 3 anos e 4 meses \\
PE & $1.996 \mathrm{~kg} /$ ano & $1.996 \mathrm{~kg} / \mathrm{ano}$ & $1.996 \mathrm{~kg} / \mathrm{ano}$ & $1.996 \mathrm{~kg} / \mathrm{ano}$ \\
\hline
\end{tabular}

\section{Cenário 02 - Ano 2017}

Com os valores obtidos no fluxo de caixa de 2017, foi possível encontrar os resultados dos indicadores de viabilidade econômica apresentados na Tabela 2, que comprovaram a viabilidade econômica e rentabilidade da produção de pitaya fertirrigada em Tomé-Açu, sendo que estão destacados os seguintes resultados: da TIR, que foi mais que $25 \%$, e do VPL, sob a taxa de $10 \%$, que foi de $\mathrm{R} \$ 132.972,51$, confirmando a rentabilidade da pitaya neste sistema, apesar das oscilações de preços corridas neste ano.

Tabela 2: Resultado dos indicadores de viabilidade com os custos de produção do ano de 2017.

\begin{tabular}{|c|c|c|c|c|}
\hline \multicolumn{5}{|c|}{ Taxas de financiamento } \\
\hline Cenário 02 - 2017 & $2,0 \%$ a.a. & $5,0 \%$ a.a. & $5,5 \%$ a.a. & 10,0\% a.a. \\
\hline VPL & $R \$ 325.726,33$ & $R \$ 234.303,50$ & $\mathrm{R} \$ 221.714,45$ & $R \$ 132.972,51$ \\
\hline IBC & $\mathrm{R} \$ 4,90$ & $\mathrm{R} \$ 3,81$ & $\mathrm{R} \$ 3,66$ & $\mathrm{R} \$ 2,59$ \\
\hline TIR & $25,26 \%$ & $25,26 \%$ & $25,26 \%$ & $25,26 \%$ \\
\hline PayBack & 4 anos e 5 meses & 4 anos e 5 meses & 4 anos e 5 meses & 4 anos e 5 meses \\
\hline $\mathrm{PE}$ & $2.649 \mathrm{~kg} / \mathrm{ano}$ & $2.649 \mathrm{~kg} / \mathrm{ano}$ & $2.649 \mathrm{~kg} / \mathrm{ano}$ & $2.649 \mathrm{~kg} / \mathrm{ano}$ \\
\hline
\end{tabular}

\section{Cenário 03 - Ano 2018}

Os resultados dos indicadores de viabilidade para o fluxo de caixa do ano de 2018 mostram que, apesar de ter atingido o menor preço de venda e a maior elevação dos custos comparado aos demais cenários, ainda assim a produção de pitaya fertirrigada apresenta uma rentabilidade satisfatória. De acordo com os resultados, o PE foi de $3.347 \mathrm{~kg}$, o qual determina a necessidade de produção e venda para que o fluxo de caixa se torne nulo, ou seja, a quantidade necessária para cobrir todos os custos. Portanto, esse resultado fica muito abaixo do pico de produção, que é de 16.000 kg de pitaya após se atingir a estabilidade produtiva, resultado este que representa uma margem de segurança considerável ao produtor. 
Tabela 3: Resultado dos indicadores de viabilidade com os custos de produção do ano de 2018.

\begin{tabular}{|c|c|c|c|c|}
\hline \multicolumn{5}{|c|}{ Taxas de Financiamento } \\
\hline Cenário 03 - 2018 & $2,0 \%$ a.a. & $5,0 \%$ a.a. & $5,5 \%$ a.a. & 10,0\% a.a. \\
\hline VPL & $\mathrm{R} \$ 227.599,80$ & $\mathrm{R} \$ 154.393,10$ & $\mathrm{R} \$ 144.320,12$ & $\mathrm{R} \$ 73.383,50$ \\
\hline IBC & $R \$ 3,41$ & $\mathrm{R} \$ 2,63$ & $R \$ 2,53$ & $\mathrm{R} \$ 1,78$ \\
\hline TIR & $18,32 \%$ & $18,32 \%$ & $18,32 \%$ & $18,32 \%$ \\
\hline PayBack & 5 anos e 5 meses & 5 anos e 5 meses & 5 anos e 5 meses & 5 anos e 5 meses \\
\hline $\mathrm{PE}$ & $3.347 \mathrm{~kg} / \mathrm{ano}$ & $3.347 \mathrm{~kg} / \mathrm{ano}$ & $3.347 \mathrm{~kg} / \mathrm{ano}$ & 3.347 kg/ano \\
\hline
\end{tabular}

\section{Cenário 04 - Ano 2019}

Com as estimativas de fluxo de caixa para 2019, percebe-se que a produção de pitaya seguirá sendo viável economicamente, com resultados positivos em todos os indicadores calculados, como pode ser visto na Tabela 4. O cenário para 2019, confirma-se com um IBC de $R \$ 2,85$ bruto para cada unidade monetária investida, baseado em alta taxa de financiamento (10\% a.a.). A TIR igual a $28,15 \%$ atesta, assim, a capacidade de pagamento de compromissos e geração de receita ao produtor, no qual alcança o retorno de seus investimentos após quatro anos de produtividade, firmando a continuação de rentabilidade da cultura para o cenário estimado.

Tabela 4: Resultado dos indicadores de viabilidade com os custos de produção do ano de 2019.

\begin{tabular}{lllll}
\hline Taxas de Financiamento & & & & \\
Cenário 04 - 2019 & $2,0 \%$ a.a. & $5,0 \%$ a.a. & $5,5 \%$ a.a. & $10,0 \%$ a.a. \\
\hline VPL & $\mathrm{R} \$ 371.322,21$ & $\mathrm{R} \$ 271.142,14$ & $\mathrm{R} \$ 257.336,97$ & $\mathrm{R} \$ 159.916,72$ \\
$\mathrm{IBC}$ & $\mathrm{R} \$ 5,28$ & $\mathrm{R} \$ 4,13$ & $\mathrm{R} \$ 3,97$ & $\mathrm{R} \$ 2,85$ \\
TIR & $28,15 \%$ & $28,15 \%$ & $28,15 \%$ & $28,15 \%$ \\
PayBack & 4 anos & 4 anos & 4 anos & 4 anos \\
PE & $2.516 \mathrm{~kg} / \mathrm{ano}$ & $2.516 \mathrm{~kg} / \mathrm{ano}$ & $2.516 \mathrm{~kg} / \mathrm{ano}$ & $2.516 \mathrm{~kg} / \mathrm{ano}$ \\
\hline
\end{tabular}

\section{Análise de Sensibilidade}

Sobre a análise de sensibilidade, apresentada na Figura 2, considerando as oscilações dos custos de produção e preços de venda ocorridas no período pesquisado de 2016 a 2018 e mais o valor projetado para o cenário de 2019, obteve-se as oscilações dos indicadores de viabilidade. Desse modo, foi proporcionada uma redução na atratividade da cultura avaliada, apesar de continuar sendo viável. Mostrando a variação ocorrida do Cenário 01 para o Cenário 04, sucedeu uma retração de $\mathrm{R} \$ 55.016,85$ no VPL, o IBC reduziu para $\mathrm{R} \$ 2,85$, apresentando diferença de $\mathrm{R} \$ 1,23$, e, do mesmo modo, a TIR caiu de $36 \%$ para $28 \%$ na comparação entre os cenários, quando analisados a uma taxa de financiamento de $10 \%$ a.a.

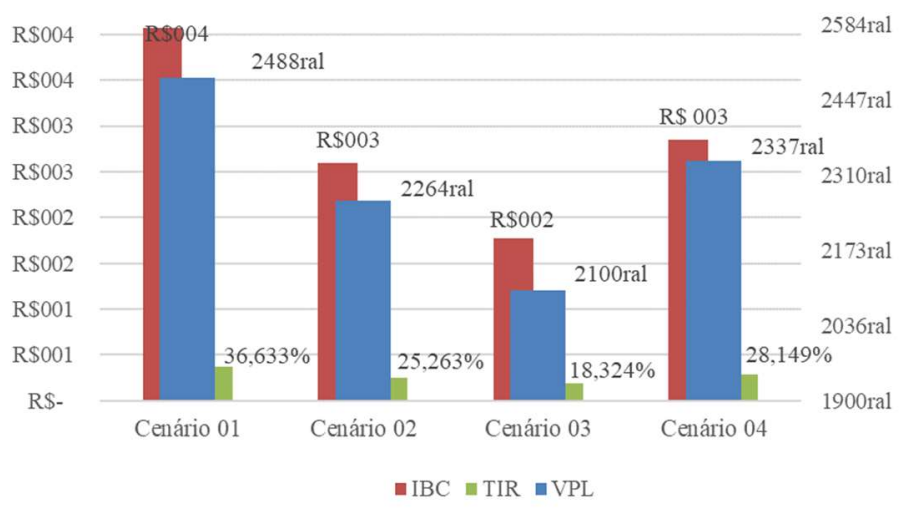

Figura 2: Variação dos indicadores de viabilidade econômica. 
Analisando a sensibilidade, todos os indicadores mostraram-se viáveis, ou seja, o VPL apresentou resultado positivo em ambos os cenários, o IBC > 1 e a TIR > Taxa Mínima de Atratividade (considerada de $6 \%$ a.a.). Contudo, apesar dos gastos com insumos serem elevados e consistirem em uma preocupação para o produtor, o aumento dos custos apresentou baixa sensibilidade. Entretanto, as alterações no preço de venda refletem diretamente nas receitas, retraindo significativamente o resultado a ser obtido ao final do projeto ou vida útil do plantio.

A retração de cerca de $26 \%$ no VPL é decorrente da queda de $9 \%$ no preço pago no principal canal de comercialização (CAMTA) e ainda da elevação dos custos totais em $46 \%$ no período pesquisado, e de $1 \%$ apenas o cenário projetado para o ano de 2019. Simulando a manutenção do preço do ano de 2016 - R\$4,00 —, em 2019, esse percentual de queda no VPL ficaria em 8\%. Portanto, os produtores rurais precisam estar atentos à dinâmica do mercado, preocupando-se sempre com as oscilações dos preços e outros indicadores que podem ou não determinar a continuidade dos investimentos (SANTANA, 2005).

A TIR de 28,15\% considera um indicador importante, pois demonstrou que esse é o montante máximo de juros de financiamento ou outra fonte de recurso que o projeto suportaria pagar, ao mesmo tempo que confirma um retorno percentual considerável em relação ao investimento inicial simulado, que seria de $\mathrm{R} \$ 86.667,55$ no último cenário para 2019.

Caso a TIR se igualasse ao percentual da taxa mínima de atratividade que é de 6\% a.a., o produtor seria indiferente quanto à opção de investir na cultura. No entanto, como a TIR do último cenário simulado resultou em mais de $20 \%$ acima da TMA, ou seja, mais que o triplo, a decisão acerca do investimento ainda se mostra atrativa devido ao potencial de retorno, apesar das oscilações de preços ocorridas.

Dessa maneira, é importante colocar que, apesar da oscilação dos custos e preços, todos os indicadores mostram que a cultura é viável, principalmente pela probabilidade de comercialização. Portanto, a tendência é o crescente aumento da produção para os anos posteriores. Assim, confirma-se que a cultura pode ser uma relevante fonte de renda para os produtores e uma potencial oportunidade de investimento a novos empreendedores.

\section{CONCLUSÕES}

Por intermédio do estudo realizado, é possível afirmar que a implantação de áreas de cultivo de pitaya fertirrigada é viável economicamente e apresenta sensibilidade considerável, principalmente ao preço de comercialização, que muda tanto de um ano para o outro quanto de um canal de comercialização para o outro. Porém, é importante salientar que, em todos esses cenários, todos os indicadores de viabilidade mostraram resultados positivos, o que caracteriza o cultivo da pitaya fertirrigada como um investimento de risco aceitável.

\section{REFERÊNCIAS}

ABREU, W. C.; LOPES, C. O.; PINTO, K. M.; OLIVEIRA, L. A.; CARVALHO, G. B. M.; BARCELO, M. P.. Características físicoquímicas e atividade total de pitaias vermelha e branca.
Revista Instituto Adolfo Lutz, v.71, n.1, p.656-661, 2012.

ASSAF NETO, A.. Finanças Corporativas e Valor. São Paulo: 
Atlas, 2010.

BASTOS, D. C.; PIO, R.; SCARPARE, J. F.; LIBARDI, M. N.; ALMEIDA, F. P.; GALUCHI, T. D.; BAKKER, S. T.. Propagação da Pitaya 'vermelha' por estaquia. Ciência e Agrotecnologia, v.30, n.6, p.1106-1109, 2006. DOI: http://doi.org/10.1590/S1413-70542006000600009

BROM, L. G.; BALIAN, J. E.. Análise de investimentos e capital de giro: Conceitos e aplicações. São Paulo: Saraiva, 2007.

BURT, C. M.; O'CONNOR, K.; RUEHR, T.. Fertigation. San Luis Obispo: Irrigation Training \& Research Center, 1995.

CAMTA. Relatório de Comercialização: ano 2017. Diretoria Comercial da Cooperativa Agrícola Mista de Tomé-Açu. Tomé-Açu: CAMTA, 2018.

CANTO, A. R.; ALBARADO, J. G.; SANTAROSA, M. G.; RAMOS, C. J.; GARCÍA, M. M.; HERNÁNDEZ, L. P.. El cultivo de pitahaya en Yucatan. Yucatán: Universidad Autonoma Chapingo, 1993.

CAVALCANTE, Í. L.; BECKMANN, M. Z.; MARTINS, A. B.; CAVALCANTE, L. F.. Pitaia e a salinidade. In: CAVALCANTE, L. F.; LIMA, E. M.. Algumas Frutíferas Tropicais e a Salinidade. Jaboticabal: FUNEP, 2006. p.137-148.

CLEMENTE, A.. Projetos empresariais e públicos. São Paulo: Atlas, 1998.

CRUZ, J. A. M.; RODRÍGUEZ-LARRAMENDI, L.; ORTIZ-PÉREZ, R.; FONSECA-FLORES, M. A.; RUÍZ, H. G.; GUEVARAHERNÁNDEZ, F.. Revisión bibliográfica. Pitahaya (Hylocereus spp.) un recurso fitogenético con historia y futuro para el trópico seco mexicano. Cultivos Tropicales, v.36, n.1, p.6776, 2015

DONADIO, L. C.. Pitaya. Rev. Bras. Frutic., v.31, n.3, p.637929, 2009. DOI: http://doi.org/10.1590/S010029452009000300001

GARCÍA-RUBIO, L. A.; VARGAS-PONCE, O.; RAMÍREZMIRELES, F. J.; MUNGUÍA-LINO, G.; CORONA-OCEGUERA, C. A.; CRUZ-HERNÁNDEZ, T.. Distribución geográfica de Hylocereus (Cactaceae) en México. Botanical Sciences, v.93, n.4, p.921-939, 2015. DOI:

http://doi.org/10.17129/botsci.282

GITMAN, L. J.. Princípios de administração financeira. São Paulo: Pearson Pretice Hall, 2010.

GITMAN, L. J.. Princípios de administração financeira. São Paulo: Harbra, 1999.

GUNASENA, H. M.; PUSHPAKUMARA, D. G.; KARIYAWASAM, M.. Dragon Fruit-Hylocereus undatus (Haw.) Britton and Rose. In: PUSHPAKUMARA, D. G.; GUNASENA, H. M.; SINGH,
V. P.. Underutilized fruit trees in Sri Lanka. New Delhi: India World Agroforestry Centre, 2007. p.110-142.

JUNQUEIRA, K. P.; JUNQUEIRA, N. V.; RAMOS, J. D.; PEREIRA, A. V.. Informações preliminares sobre uma espécie de pitaya do Cerrado. Planaltina: Embrapa Cerrados, 2002.

LE BELLEC, F.; VAILLANT, F.; IMBERT, E.. Pitahaya (Hylocereus spp.): a new fruit crop, a market with a future. Fruits, v.61, n.4, p.237-250, 2006. DOI:

http://doi.org/10.1051/fruits:2006021

LOUREIRO, J. P.; GONÇALVES, C. M.; SARGES, D. B.; ROCHA, J. T.; FRAZÃO, J. C.; SANTOS, L. S.. Comparação sobre a viabilidade econômica de sistemas de produção de hortaliças hidropônicas com diferentes níveis de tecnologia, nos municípios de Concórdia do Pará e Tomé-Açu-PA. BJD, v.5, n.11, p.24607-24621, 2019. DOI: http://doi.org/10.34117/bjdv5n11-140

MARQUES, V. B.; RAMOS, J. D.; ARAÚJO, N. A.; MOREIRA, R. A.. Custo de produção e rentabilidade na cultura da pitaya sob o efeito de adubação orgânica. Científica, v.40, n.2, p.138-149, 2012. DOI: http://doi.org/10.15361/19845529.2012v40n2p138+-+149

MIZRAHI, Y.; NERD, A.; NOBEL, P. S.. Cacti as crops. Horticultural Reviews, v.18, n.1, p.291-320, 1997. DOI: http://doi.org/10.1002/9780470650608.ch6

NUNES, E. N.; SOUSA, A. B.; LUCENA, C. M.; SILVA, S. M.; LUCENA, R. P.; ALVES, C. B.. Pitaia (Hylocereus sp.): Uma revisão para o Brasil. Gaia Scientia, v.8, n.1, p.90-98, 2014.

PONCIANO, N. J.; SOUZA, P. M.; MATA, H. T.; VIEIRA, J. R.; MORGADO, I. F.. Análise de viabilidade econômica e de risco da fruticultura na região norte Fluminense. RESR, v.42, n.4, p.615-635, 2004. DOI: http://doi.org/10.1590/S010320032004000400005

SANTANA, A. C.. Elementos de economia, agronegócio e desenvolvimento local. Belém: UFRA, 2005.

SATO, S. A.; RIBEIRO, S. A.; SATO, M. K.; SOUZA, J. S. Caracterização física e físico-química de pitayas vermelhas (Hylocereus costaricensis). Journal of Bioenergy and Food Science, v.1, n.2, p.46-56, 2014.

SILVA, M. Z.; SCARPIN, J. E.; ROCHA, W.; DOMENICO, D. D. Fatores contingenciais que contribuem para a decisão de modificação do sistema de custeio: estudo de caso em uma indústria moageira. RAUSP, v.49, n.2, p.267-279, 2014. DOI: https://doi.org/10.5700/rausp1145

VAILLANT, F.; PEREZ, A.; DAVILA, I.; DORNIER, M.; REYNES, M.. Colorant and antioxidant properties of red pitahaya (Hylocereus sp.). Fruits, v.60, n.1, p.1-7, 2005. DOI: http://doi.org/10.1051/fruits:2005007

A CBPC - Companhia Brasileira de Produção Científica (CNPJ: 11.221.422/0001-03) detém os direitos materiais desta publicação. Os direitos referem-se à publicação do trabalho em qualquer parte do mundo, incluindo os direitos às renovações, expansões e disseminaç̃̃es da contribuição, bem como outros direitos subsidiários. Todos os trabalhos publicados eletronicamente poderão posteriormente ser publicados em coletâneas impressas sob coordenação da Sustenere Publishing, da Companhia Brasileira de Produção Científica e seus parceiros autorizados. Os (as) autores (as) preservam os direitos autorais, mas não têm permissão para a publicação da contribuição em outro meio, impresso ou digital, em português ou em tradução. 